\title{
Multi-resolution shape-based image retrieval using Ridgelet transform
}

\begin{abstract}
Complicated shapes can be effectively characterized using multi-resolution descriptors. One popular method is the Ridgelet transform which has enjoyed very little exposure in describing shapes for Content-based Image Retrieval (CBIR). Many of the existing Ridgelet transforms are only applied on images of size $\mathrm{M} \times \mathrm{M}$. For $\mathrm{M} \times \mathrm{N}$ sized images, they need to be segmented into $\mathrm{M} \times \mathrm{M}$ sub-images prior to processing. A different number of orientations and cut-off points for the Radon transform parameters also need to be utilized according to the image size. This paper presents a new shape descriptor for CBIR based on Ridgelet transform which is able to handle images of various sizes. The utilization of the ellipse template for better image coverage and the normalization of the Ridgelet transform are introduced. For better retrieval, a template-option scheme is also introduced. Retrieval effectiveness obtained by the proposed method has shown to be higher compared to several previous descriptors.
\end{abstract}

Keyword: Content-based image retrieval; Multi-resolution; Ridgelet transform; Shape descriptor 